\title{
Oral Contraceptives: Mind-Body Poison
}

\section{Brogan $\mathbf{K}^{*}$}

ABIHM, 280 Madison Ave 702 New York, USA

The modern woman considers synthetic hormone replacement a right; however, this entitlement comes at arguable social and psycho physiologic costs including tacit permissiveness toward reckless unprotected sex, the wholesale delegation of contraception to the female counterpart, and the fundamental divorce of a woman from the very feedback systems that fire up her reproductive age vitality. With over 100 million women using this form of hormonal suppression worldwide, few have likely considered the subtle but important perturbations to the system, not to mention the consensus risks of thromboembolism, hypertension, cerebrovascular events, gallstones, and cancer.

As notorious as our hormones are for wreaking havoc, they are what pop us into high relief-they excite us, move us, drive us and enliven us. The highly non-linear relationships between sex hormones, thyroid hormone, and adrenal hormones are like the magic of 3-D glasses: if you cover one lens, things just don't look as exciting.

When patients come to me with complaints of low libido, low or flat mood, weight gain, hair loss, and cloudy thinking, one of my first questions is "Are you on the Pill?" When they come complaining about premenstrual irritability, insomnia, tearfulness, bloating, and breast tenderness, requesting that I sanction beginning a course of oral contraceptives and perhaps an antidepressant, the one-size-fitsall-cure-all of psychiatrists and gynecologists nationwide, my first comment is "There's a better way." The signs of hormonal imbalance are often quickly dismissed as a problem too complex to rectify from its source. In my practice, I first look at diagnostics to better personalize dietary recommendations, targeted nutritional interventions, and herbal options to promote the body's own drive toward harmony. If it's not enough to celebrate their right to an optimally functioning hormonal system, and they also want to preserve their right not to have a fertilized egg, then I recommend a non-hormonal IUD, nifty gadgets like the Pearly [1], or the good old' fashioned condom.

Why is it that I think rendering a woman a 2-dimensional version of her hormonal self is such a big deal? Simply because, if she's in my office, she is someone who has struggled with mood and anxiety, and the last thing she should be doing is stacking the cards against her recovery with synthetic hormones and the pharmacologic burden they bring. Since the 1960s, there has been controversy around the potential mood effects of oral contraceptives, but over 50 years of their use has not settled the question. There is acknowledgement; however, that depression is the most common reason for discontinuation of use. I don't need pilot studies [2] that demonstrate women using the combined oral contraceptive pill were significantly more depressed than a matched group who were not, to persuade me because I see it first hand, most notably, in those who begin contraception (hormonal IUD, progestin-only, or combined) postpartum.

The data, overall, is compromised of inconsistent assessments of mood pathology across the cycle and between groups, and often by study design, but suggests that there is likely a subset of women for whom oral contraceptives represent a major risk factor for depression and/or related mood disturbance. Who might these women be? From 13 prospective trials [3], it appears that they have a personal or family psychiatric history (though doesn't that include the entire population at this point?), one that has been exacerbated by pregnancy/postpartum, and premenstrual periods, and young age. More specifically, women with premenstrual mood symptoms prior to use experience more adverse effects with lower progestin dosages or triphasic OCs, unlike women without this history who experience more psychiatric side effects with higher progestin preparations.

Could these side effects be just coincidence? Could they represent "confounding by indication" or the fact that many women who opt to suppress their cycle might already be prone to depression? It's possible, but so are some of these important mechanistic insights:

- Synthetic hormones like those in combined oral contraceptives increase thyroid and sex hormone binding globulin, effectively decreasing the available testosterone and thyroid hormone in circulation (which can render you of nun-like libido, and functionally hypothyroid or depressed, constipated, overweight, cloudy, with dry skin and hair, to boot!). A randomized, open-label, 9 week trial [4] of 3 forms of hormonal contraception found that they all increased SHBG, as well as insulin resistance, and markers of inflammation such as c-reactive protein. Another study [5] suggests that increases in SHBG may persist long after discontinuation of the Pill contributing to sexual dysfunction/low libido. Incidentally, endocrine modulators such as PCBs, BPA, and pthalates, as well as poor excretion of estrogen as occurs with gut dysbiosis, can also contribute to elevated SHBG and lead to undesirable states of "estrogen dominance".

- Oral contraceptives promote oxidative stress. Stress is often defined as the inability to cope with demands, and oxidative stress is a destructive force in the body perpetuated by reactive oxygen species that outnumber available antioxidant enzymes and factors. One measure of oxidative stress, lipid peroxidation, was found [6] to be higher in those taking OCs, and improved (not quite to baseline controls) when they were treated with vitamins $\mathrm{E}$ and $\mathrm{C}$, known antioxidants. Thus OCs may decrease the activity of two major defense systems in the body.

- Oral contraceptives deplete vitamins, minerals, and antioxidants as measured [7] by activity of Coenzyme Q10, vitamin E, and Total Antioxidant Activity. More specifically, OCPs have been acknowledged to deplete vitamin $\mathrm{B} 6$, a cofactor for the production of serotonin and GABA, as well as zinc, selenium, phosphorus, and magnesium demonstrated by this cross-sectional randomized study [8]. Interestingly, elevated levels of copper (which can upregulate catecholamines, potentially causing feelings of overstimulation), iron (which can be a pro-oxidant), calcium, and cadmium were elevated compared to controls. Given that replacing and correcting these

*Corresponding author: Kelly Brogan, ABIHM, 280 Madison Ave 702 New York, NY 10016, USA, E-mail: drbrogan@kellybroganmd.com

Received June 03, 2013; Accepted June 14, 2013; Published June 17, 2013

Citation: Brogan K (2013) Oral Contraceptives: Mind-Body Poison. Altern Integ Med 2: 124. doi:10.4172/2327-5162.1000124

Copyright: () 2013 Brogan K. This is an open-access article distributed under the terms of the Creative Commons Attribution License, which permits unrestricted use, distribution, and reproduction in any medium, provided the original author and source are credited. 
vitamins can be an imperfect effort, perhaps its best not to mess with them in the first place!

The review [3] also discusses that the identification of patients vulnerable to these risks should include assessment of hormone sensitivity (menstrual cycle history, acne/hirsuitism, body mass index), comorbid diagnoses indicative of hormonal disarray (thyroid, diabetes, breast cancer), and baseline hormone assessment. I would add that an assessment of antioxidant and micronutrient status and markers of inflammation would also help to risk stratify. This tends to be my major concern with regard to pharmaceutical interventions - there's just no free lunch with medication treatment, and a risk/benefit analysis is very difficult to do if we don't know what environmental and genetic risks an individual is bringing to the table. If there is a treatment option that presents minimal to no appreciable risks and some degree of evidencebased benefit, this, to me would represent the kinder, gentler road to health. Some of these interventions might include dietary modification, stress management, exercise, magnesium, B6, maca, evening primrose oil, and chaste tree. These days, women's lib looks a lot more like a healthy, happy menstrual cycle free from the grips of a prescription.

\section{References}

1. The Pearly Monitor.
2. Kulkarni SK, Bhutani MK, Bishnoi M (2008) Antidepressant activity of curcumin involvement of serotonin and dopamine system. Psychopharmacology (Berl) 201: 435-442.

3. Oinonen KA, Mazmanian D (2002) To what extent do oral contraceptives influence mood and affect? J Affect Disord 70: 229-40.

4. Piltonen T, Puurunen J, Hedberg P, Ruokonen A, Mutt SJ, et al. (2012) Oral, transdermal and vaginal combined contraceptives induce an increase in markers of chronic inflammation and impair insulin sensitivity in young healthy normal-weight women: a randomized study.Hum Reprod 27: 3046-3056.

5. Panzer C, Wise S, Fantini G, Kang D, Munarriz R, et al. (2006) Impact of oral contraceptives on sex hormone-binding globulin and androgen levels: a retrospective study in women with sexual dysfunction. J Sex Med 3: 104-113.

6. Zal F, Mostafavi-Pour Z, Amini F, Heidari A (2012) Effect of vitamin E and C supplements on lipid peroxidation and GSH-dependent antioxidant enzyme status in the blood of women consuming oral contraceptives. Contraception 86: 62-66.

7. Palan PR, Strube F, Mikhail MS (2010) Effects of Oral, Vaginal, and Transdermal Hormonal Contraception on Serum Levels of Coenzyme Q10, Vitamin E, and Total Antioxidant Activity. Obstet Gynecol Int 2010: 925635.

8. Akinloye O, Adebayo TO, Oguntibeju OO, Oparinde DP, Ogunyemi EO (2011) Effects of contraceptives on serum trace elements, calcium and phosphorus levels. West Indian Med J 60: 308-315. 